\title{
RADIO, OPTICAL AND X-RAY EMISSION FROM PULSARS
}

\author{
B. BERTOTTI \\ European Space Research Institute (ESRIN), Frascati, Rome \\ A. CAVALIERE \\ Laboratorio Gas Ionizzati (EURATOM-CNEN), Frascati, Rome \\ and \\ F. PACIN I \\ Laboratorio di Astrofisica, Frascati, Rome
}

\begin{abstract}
We examine the electromagnetic spectrum of the Crab Nebula pulsar NP 0532. The present observations are in rough agreement with the idea that the orbit of the radiating particles involves two different radii of curvature. One of the possible models requires electrons with a typical energy of the order of $100 \mathrm{MeV}$ and a density $\approx 10^{9} \mathrm{~cm}^{-3}$. A correlation length of about $10 \mathrm{~cm}$ could then give the coherence of the radio emission. Slow pulsars are unlikely to emit an appreciable amount of optical or X-ray radiation but a weak infrared emission is possible.
\end{abstract}

1. We can now be reasonably confident that the timing and the basic energy source of pulsars are both related to the rotation of a magnetic neutron star (Gold, 1968; Pacini, 1968). This confidence is based upon the observed repetition rate which is consistent with the neutron star idea but in most cases exceeds the Jeans frequency of a white dwarf. Furthermore, the period increase observed in the case of several pulsars can be explained as loss of rotational energy (Gold, 1968; Pacini, 1968; Gold, 1969; Finzi and Wolf, 1969; Bertotti et al., 1969a; Gunn and Ostriker 1969a), but it cannot be easily interpreted in a vibrating model. In the particular case of the Crab Nebula pulsar NP 0532, the loss of rotational energy is of the same order as the total energy requirement of the Crab Nebula, about $10^{38} \mathrm{ergs} \mathrm{sec}^{-1}$ (largely under form of relativistic particles). It appears therefore that the rotation of a magnetic neutron star is connected not only to the pulsar phenomenon, but also to the overall high energy activity of Supernovae Remnants (Pacini, 1967, 1968; Gold, 1969; Bertotti et al., 1969a; Gunn and Ostriker, 1969a, b; Finzi and Wolf, 1969).

An understanding of pulsars requires also a description of the interaction between the spinning star and the surrounding plasma, and furthermore an analysis of the radiation process.

Several suggestions have been put forward concerning the first of these points, namely how rotational energy can be released from the star. These involve:

(a) rigid corotation of the magnetosphere up to a critical distance, limited by $R=c / \Omega$, where $\Omega$ is the angular rotation frequency of the star (Gold, 1968, 1969; Goldreich and Julian, 1969; Michel and Tucker, 1969; Michel, 1969);

(b) emission of electromagnetic energy from an oblique rotator, which, for distances $r$ greater than $c / \Omega$, has the character of magnetic dipole-radiation (Pacini, 1967, 1968; Bertotti et al., 1969a; Gunn and Ostriker, 1969a, b).

The radiation processes which have been suggested are essentially induced scattering 
from relativistic particles (Ginzburg et al., 1968, 1969; Bertotti et al., 1969a ; Cavaliere, 1969) and synchrotron-like radiation (Gold, 1968, 1969; Michel and Tucker, 1969). These processes seem to be good candidates to account for the high radio brightness, broad band and polarization of the pulsar radiation.

On the observational side, while most sources appear to emit only in the radio band, the recent discovery of optical (Cocke et al., 1969) and X-ray (Bradt et al., 1969; Fritz et al., 1969; Haymes et al., 1969) flashes from NP 0532 extends, in this particular case, the spectral band to be accounted for by the emission mechanisms.

In this paper, we shall concentrate upon the electromagnetic spectrum of NP0532 and examine a simple synchrotron-like radiation process based indeed upon the vectorial acceleration of relativistic particles with $\gamma=E / m c^{2} \gg 1$ (See also Bertotti et al., 1969b).

Our main assumption will be that the overall kinematics of relativistic particles around the star entails two widely different radii of curvature $\varrho$ and hence two fundamental frequencies $c / \varrho$ : these qualitative features result in a number of detailed models. Here we shall concentrate mainly upon the emission which results, at $r \geqslant c / \Omega$, from electrons forced to rotate at the basic frequency $\Omega$ by the stellar electromagnetic field and also circling around the lines of force of the local magnetic field $B$. The first motion can be due to the well-known Gold's idea of corotation up to the speed of light cylinder $R=c / \Omega$; alternatively, outside $R$, it can be forced upon by the absorption of the angular momentum carried by the magnetic dipole radiation emitted from the star. For NP0532 we have $\Omega=200 \mathrm{sec}^{-1}$. On the other hand, the magnetic field at distances $\approx c / \Omega$ must be about $10^{6} \mathrm{G}$ (corresponding to a surface field of about $10^{12} \mathrm{G}$ ) if the energetic stockpile of the Crab Nebula is to be drawn from the stellar rotation through the agency of the magnetic field. This would imply a gyrofrequency in the rest frame $\omega_{L}=e B / m c$ much larger than the frequency $\Omega$.

We note explicitly that the same qualitative considerations would be relevant even if the motion has a torsion and a tangential acceleration. In particular, they are valid in the case of emission of particles from the polar regions (Goldreich and Julian, 1969; Radhakrishnan and Cooke, 1969), provided that the particles simultaneously move away from the poles along the field lines and also circle around them. (We thank Dr. M. Rees for calling our attention on this point.)

In the following, we shall minimize the number of free parameters by taking a small dispersion in the particles energy, i.e. $\Delta \gamma / \gamma \ll 1$. Fit to detailed, forthcoming spectra may require relaxing this constraint in a second approximation: for the time being, we consider powers emitted across large spectral regions and we recall that the radio spectrum of NP 0532 has a peak about $100 \mathrm{MHz}$ (Comella et al., 1969) while the optical spectrum is roughly flat and shows an ultraviolet excess (Cocke et al., 1969).

2. We recall briefly the emission characteristics of the motion of relativistic particles (for a more detailed account, see, e.g. Ginzburg and Syrovatskii, 1965, 1969). For these particles, the high frequency features of the radiation depend essentially upon the radius of curvature $\varrho$ (or the associated angular frequency $\bar{\Omega}=c / \varrho$ ) at those points 
of the orbit whose tangent lies within an angle $1 / \gamma$ with the visual line of the observer (for single particles the peak emission is indeed concentrated within a cone of width $1 / \gamma)$.

The spectrum is composed of harmonics of the fundamental frequency $\bar{\Omega}$; this structure is irrelevant for $\omega \gg \bar{\Omega}$, and the corresponding spectral power $p(\omega)$ can be approximated as follows

$$
\begin{aligned}
& p(\omega) \approx \frac{e^{2}}{c} \bar{\Omega} \gamma\left(\begin{array}{c}
\omega \\
\omega_{\mathrm{cr} .}
\end{array}\right)^{1 / 3} \text { for } \omega \lesssim \omega_{\mathrm{cr} .} \\
& p(\omega) \approx \frac{e^{2}}{c} \bar{\Omega} \gamma\left(\frac{\omega}{\omega_{\mathrm{cr} .}}\right)^{1 / 2} \exp \left(-\frac{\omega}{\omega_{\mathrm{cr} .}}\right) \text { for } \omega>\omega_{\mathrm{cr} .}
\end{aligned}
$$

where the critical frequency is given by $\omega_{\mathrm{cr} .}=\frac{3}{2} \bar{\Omega} \gamma^{3}$.

For the case of a helicoidal path and for the density effects, see Ginzburg and Syrovatskii (1969).

For our purposes, coherence effects are very important, as indicated by the very high brightness temperature of the pulsars radio emission. In the following we shall assume that coherence stems from bunching of particles (Gold, 1968, 1969), even if other causes are possible (see Ginzburg et al., 1968, 1969). One particular, but not exclusive, cause of correlations in the electron density can be electrostatic instability feeding on relative streaming energy.

Coherent radiation by bunches of particles with correlation length $L$ and density $n$ would give an emission per unit volume

$$
P(\omega)=p(\omega) n(1+F) .
$$

The coherence factor $F$, in the case where turbulent fluctuations have frequencies smaller than the radiation angular frequency $\omega$, is of the form

$$
F=n L^{3} f(c / \omega)
$$

Here $f(c / \omega)$ is the dimensionless Fourier transform of the density correlation function and vanishes for $L \gg c / \omega$. For a gaussian bunching, for example, we obtain

$$
f(c / \omega)=\exp \left[-\left(\frac{L \omega}{4 \pi c}\right)^{2}\right]
$$

but different and weaker dependences upon the frequency are conceivable.

3. We shall now discuss two different possibilities:

(a) all the observed spectrum is associated with the basic frequency $\Omega$ (the gyrofrequency could then give rise to a high-energy $\gamma$-rays emission);

(b) the radiospectrum is associated with the frequency $\Omega$ while the optical and the $\mathrm{X}$-ray emission are related to the gyrofrequency. 
We shall find that the first possibility implies much higher values for the particles energy and for their density than the second one.

Let us investigate first the case where the entire electromagnetic spectrum observed up to now is connected with the basic angular frequency $\Omega$. It is then easy to show that the observed ratio of power in the optical and X-ray band (respectively indicated by $\Delta \omega_{0} \approx 5 \times 10^{15} \mathrm{sec}^{-1}$ and by $\Delta \omega_{x} \approx 10^{19} \mathrm{sec}^{-1}$ ), fixes the critical frequency $\omega_{1}=\frac{3}{2} \Omega \gamma^{3}$. The relation

$$
\frac{P_{0}}{P_{x}} \approx 10^{-2} \approx\left(\frac{\omega_{0}}{\omega_{1}}\right)^{1 / 3}\left(\frac{\omega_{1}}{\omega_{x}}\right)^{1 / 2} \frac{\Delta \omega_{0}}{\Delta \omega_{x}} \exp \left(-\frac{\omega_{x}}{\omega_{1}}\right)
$$

gives then, roughly, $\omega_{1} \approx 10^{18} \mathrm{sec}^{-1}$ and implies $\gamma \approx 3 \times 10^{5}$. We stress, however, that the exponential in Equation (6) follows from $\Delta \gamma / \gamma \ll 1$.

We can now determine an average coherence factor $F$ over the radio band $\left(\Delta \omega_{R} \approx 10^{9} \mathrm{sec}^{-1}\right)$ from the observed ratio

$$
P_{P_{0}}^{P_{R}} \approx 10^{-2} \approx F\left(\begin{array}{c}
\omega_{R} \\
\omega_{0}
\end{array}\right)^{1 / 3} \Delta \omega_{R} \Delta \omega_{0} .
$$

We obtain $F \approx 5 \times 10^{6}$.

The average radio power $P_{R}=\varepsilon 10^{31} \mathrm{ergs} \mathrm{sec}^{-1}$ tells us how many particles $n V$ are emitting the observed radiation from a volume $V$

$$
P_{R} \approx e_{c}^{2} \Omega \gamma\left(\frac{\omega_{R}}{\omega_{1}}\right)^{1 / 3} n V F \Delta \omega_{R}
$$

Here $\varepsilon$ is a factor introduced to take into account the beaming of the radiation: if we assume that the pulsar can be detected from most directions, $\varepsilon$ is of the order of the ratio between the pulse length $\tau$ and the period $T$, namely $\varepsilon \approx 10^{-1}$. We then obtain $n V \approx 5 \times 10^{38}$; assuming for the emitting volume a value $\Delta R^{2}$ with $\Delta \approx 10^{7} \mathrm{~cm}$ (inferred from the existence of sub-millisecond pulse structures), the particle density turns out to be $n \approx 5 \times 10^{15} \mathrm{~cm}^{-3}$.

We now examine, with similar arguments, the other case where the radio spectrum is determined by the basic frequency $\Omega$, while the optical and the X-ray emissions are connected to the Larmor gyration and hence to the critical frequency $\omega_{2}=\frac{3}{2}\left(\omega_{L} / \gamma\right) \gamma^{3}$. The relation

$$
\frac{P_{0}}{P_{x}} \approx\left(\frac{\omega_{0}}{\omega_{2}}\right)^{1 / 3}\left(\frac{\omega_{2}}{\omega_{x}}\right)^{1 / 2} \frac{\Delta \omega_{0}}{\Delta \omega_{x}} \exp \left(-\frac{\omega_{x}}{\omega_{2}}\right)
$$

gives now $\omega_{2} \approx 10^{18} \mathrm{sec}^{-1}$. This corresponds to $B \gamma^{2} \approx 5 \times 10^{12} \mathrm{G}$.

The radio spectrum can now be decreasing either because of loss of coherence or because we are dealing with frequencies $\omega>0.3 \omega_{1}$, where $\omega_{1}$ is again the critical frequency associated with the basic rotation. In the latter case we must have $\gamma \approx 200$. The corresponding value for $B$, deduced from the previous expression $B \gamma^{2} \approx 5 \times 10^{12}$, is about $10^{6} \mathrm{G}$, in agreement with a previous independent estimate. 
The observed ratio

$$
\frac{P_{R}}{P_{0}} \approx \frac{\Omega \gamma}{\omega_{L}}\left(\frac{\omega_{2}}{\omega_{0}}\right)^{1 / 3} \frac{\Delta \omega_{R}}{\Delta \omega_{0}} F
$$

gives the numerical value for the coherence factor $F \approx 10^{13}$.

Finally, the absolute radio power gives again the number density and we obtain $n_{e} \approx 10^{9} \mathrm{~cm}^{-3}$, leading to a correlation length $L \approx 10 \mathrm{~cm}$.

4. We have not discussed here the pulsed nature of the source but only its electromagnetic spectrum. We recall however that the radiation in a fixed direction comes from particles whose velocity vector lies within an angular distance of order $1 / \gamma$ from this direction: the pulse width could therefore be a consequence of the periodical filling up (due to the rotation of the star) of such a region in phase space. For example, one could have a rotating velocity distribution of angular size $\Delta \theta \approx 2 \pi \tau / T$. If $\Delta \theta>1 / \gamma$. one expects a larger linear polarization for spectral bands close or beyond the relevant critical frequency. In our case (b), we must therefore expect more primary polarization in the radio emission than in the optical emission of NP 0532; the X-rays should again be strongly polarized. For pulsars having a smaller duty cycle $\tau / T$, we expect the eventual emergency of the polarization features of single emitters: this seems to be the case for the source CP 0328 (Clark and Smith, 1969; Cocconi, 1969).

Finally, we notice explicitly that the above emission scheme explains why optical and X-ray emissions show up for NP 0532 and not for other pulsars. Indeed, for slow pulsars, the local value of the magnetic field at distances $r \approx c / \Omega$ would be much smaller than for NP 0532, provided that the surface field on the neutron star is comparable. For slow pulsars, therefore, the peak of the gyroemission would lie in the infrared, possibly around $10^{11}$ or $10^{12} \mathrm{MHz}$. Observations in this spectral range are desirable, even if the power radiated (proportional to $B^{2}$ ) is likely to be quite low. Among the pulsars known at present, only the Vela pulsar can be expected to emit (weakly) in the optical range.

\section{References}

Bertotti, B., Cavaliere, A., and Pacini, F.: 1969a, Nature 221, 624.

Bertotti, B., Cavaliere, A., and Pacini, F.: 1969b, Nature 223, 1351.

Bradt, H., Rappaport, S., Mayer, W., Nather, F. H., Warner, B., Macfarlane, M., and Kristian, J.: 1969, Nature 222, 728.

Cavaliere, A.: 1969, 'Emission Processes in Plasma', paper given at the Pisa Meeting on Pulsars, 14-16 April.

Clark, R. R. and Smith, F. G.: 1969, Nature 221, 724.

Cocconi, G.: 1969, preprint.

Cocke, W. J., Disney, M. J., and Taylor, D. J.: 1969, Nature 221, 525.

Comella, J. M., Craft, H. D., Lovelace, R. V., and Sutton, J. M.: 1969, Nature 221, 453.

Finzi, A. and Wolf, R. A.: 1969, Astrophys. J. 155, L107.

Fritz, G., Henry, R. C., Meekins, J. F., Chubb, T. A., and Friedman, H.: 1969, Science 164, 709.

Ginzburg, V. L. and Syrovatskii, S. I.: 1965, Ann. Rev. Astron. Astrophys. 3, 297.

Ginzburg, V. L. and Syrovatskii, S. I.: 1969, Ann. Rev. Astron. Astrophys. 7, 375.

Ginzburg, V. L., Zhelezniakov, V. V., and Zaitsev, V. V.: 1968, Nature 220, 535. 
Ginzburg, V. L., Zhelezniakov, V. V., and Zaitsev, V. V.: 1969, Astrophys. Space Sci. 4, 464. Gold, T.: 1968, Nature 218, 731.

Gold, T.: 1969, Nature 221, 25.

Goldreich, P. and Julian, W. H.: 1969, Astrophys. J. 157, 869.

Gunn, J. F. and Ostriker, J. P.: 1969a, Nature 221, 454.

Gunn, J. F. and Ostriker, J. P.: 1969b, Phys. Rev. Letters 22, 728.

Haymes, R. C., Freeman, K. C., and Harnden Jr., F. R.: 1969, Astrophys. J. 156, L107.

Michel, F. C.: 1969, Phys. Rev. Letters 23, 247.

Michel, F. C. and Tucker, W. H.: 1969, Nature 223, 277.

Pacini, F.: 1967, Nature 216, 567.

Pacini, F.: 1968, Nature 219, 145.

Radhakrishnan, V. and Cooke, D. J.: 1969, Astrophys. Letters 3, 225. 